\title{
Permeability of Uniformly Graded 3D Printed Granular Media
}

$5{ }^{1}$ The University of Sydney, School of Civil Engineering, Sydney, NSW 2006, Australia.

$6 \quad{ }^{2}$ Navier, Ecole des Ponts, Univ Gustave Eiffel, CNRS, Marne-la-Vallée, France.

$7 \quad{ }^{\dagger}$ Corresponding author, Email: yixiang.gan@sydney.edu.au

9 Key Points:

- 3D printed mono-sized yet different-shaped particles of scaling morphology features from real grains are used for permeability test.

- Via more accurate area than that of the volume-equivalent sphere method, KozenyCarman shape factor is found insensitive to grains shapes.

- The Kozeny-Carman equation is modified using two compressed shape factors, i.e., 16 fractal dimension and relative roughness. 


\section{Abstract}

18 The present work explores water permeability of uniformly graded irregular grains using 3D printing with controlled shapes and fractal morphological features at low Reynold's number for viscous flow. From large amount of real 3D granular morphological data, a scaling law, in terms of fractal dimension, is found to be followed. With this universal law, sand grains with controlled fractal morphological features are generated using Spherical Harmonics, and then created using 3D printing technique for water permeability tests. A modified Kozeny-Carman equation is proposed through more accurate determination of specific area, as a function of relative roughness and fractal dimension, than approximation using the volume-equivalent sphere. By isolating the contributions from specific area, the shape coefficient is found to be insensitive to particle morphology. Through benchmarking the model prediction against experiments from both this work and past literature, we demonstrate the validity and wide applicability of the modified Kozeny-Carman equation.

\section{$30 \quad$ Plain Language Summary}

The shape of grains can influence the way how water transports inside a granular material. This study uses 3D printing technique to well control grain shapes. The grain morphology is captured through combinations of relative roughness and fractal dimension, relevant to a wide range of geomaterials. We revisit the classical permeability equation with the prior knowledge of the grain shape. It is found that the model coefficients used in the permeability equation, usually obtained through fitting against experiment measurement, do indeed contain key intrinsic morphological information.

\section{Introduction}

Estimation of permeability of porous media is of vital importance in many geophysicsrelated scientific fields, such as hydrogeology, geotechnical engineering, earth science, and petroleum engineering. As a main category of porous media (Higdon and Ford, 1996), granular materials received more attention owing to their significant prevalence in various engineering applications. Granular porous media are composed of solid matrix skeleton formed by bounded or contacting grains, and pores with narrow constraints. Natural and artificial examples are widely encountered in weakly cementitious rocks in sandstone (Sun et al., 2011), sands in geotechnical structures (Chapuis, 2004), fault gouge of seismic events (Wang et al., 2019), and shale soils around multilayered reservoirs (Zheng et al., 2018). For these media, permeability is a key parameter for describing their transport and hydro-mechanical responses. Seelheim (1880) stated that in porous media flow permeability correlates to the square value of its characteristic pore diameter. Since then, many models for predicting permeability coefficients have been proposed based on empirical relations (Hazen, 1892; Shepherd, 1989), Poiseuille capillary tubes (Carman, 1956; Mortensen et al., 2005), statistical regression (Wang et al., 2017; Feng et al., 2019) and effective hydraulic radius (Carman, 1956; Costa, 2006). Due to the complexity of pore network, it is challenging to rigorously formulate a satisfactory theoretical relation between viscous flow resistance and intrinsic geometry properties of granular porous media. As a result, most of such theoretical models start with some measurable intrinsic first-principle geometrical 
factors, including sieve-determined grading, void ratio, and porosity (Hazen, 1892; Johnson et al., 1986; Chapuis and Aubertin, 2003; Ren and Santamarina, 2018).

Among these, one of the widely adopted models is the Kozeny-Carman equation (e.g., Carrier, 2002):

$k=\frac{\gamma}{\mu} \cdot \underbrace{\frac{1}{C_{\mathrm{K}-\mathrm{C}}} \cdot \frac{1}{S_{0}{ }^{2}} \cdot \frac{\varepsilon^{3}}{(1-\varepsilon)^{2}}}_{K}$,

where $\gamma$ and $\mu$ are unit weight and viscosity of permeant, respectively, $C_{\mathrm{K}-\mathrm{C}}$ the Kozeny-Carman shape coefficient, $S_{0}$ specific surface area (i.e., surface area per unit volume of particles), $\varepsilon$ porosity, $k(\mathrm{~L} / \mathrm{T})$ the coefficient of permeability, or hydraulic conductivity, depending on both intrinsic properties of porous media and fluid, and $K\left(\mathrm{~L}^{2}\right)$ the permeability only correlated with porous structures. For water at $20^{\circ} \mathrm{C}, \frac{\gamma}{\mu}$ equals $9.93 \times 10^{4} 1 / \mathrm{cm}$ s. Intuitively, contacts between grains may result in decreasing wet area, through which water flows. However, contact area is negligible when compared with the total surface area, especially for natural rough particles (Wei et al., 2020). Accompanied with Kozeny-Carman equation (Carman, 1956), the concept of tortuosity, defined as the length ratio of effective flow path to porous sample in direction of flow, is also proposed to indicate dimensionless length of fluid element flowing through pore space (David, 1993; Ghanbarian et al., 2013). Grains with the same shapes and porosity can form rather random packings, and thus different tortuosity. Such randomness may hinder the generality of tortuosity in deterministic models to predict permeability coefficients. Although many assumptions have been implemented in the original derivation of Kozeny-Carman equation, its agreement with experimental results has been widely observed (Philipse and Pathmamanoharan, 1993; Chikhi et al., 2014). As said by Wolfgang Pauli, "God made the bulk; the surface was invented by the devil". The accurate measurement of surface area in porous media can be difficult, since many solid surfaces can exhibit multiscale features. (Barclay and Buckingham, 2009; Mollon and Zhao, 2012). With the emergent of various techniques to estimate $S_{0}$, as discussed in the review paper by Santamarina et al. (2000), a wide range of $C_{\mathrm{K}-\mathrm{C}}$ have been observed for grains of different morphology features. The value of $C_{\mathrm{K}-\mathrm{C}}$ is found to be around 5 for monodispersed spheres (Carman, 1937). For peat beds and mixture of fibrous and granular beds, the corresponding $C_{\mathrm{K}-\mathrm{C}}$ are 3.4 and 12.81, respectively (Mathvan and Viraraghavan, 1992; $\mathrm{Li}$ and $\mathrm{Gu}, 2005$ ). To exclude the effects of particle grading on estimating $C_{\mathrm{K}-\mathrm{C}}$, investigations are carried out using mono-sized aspherical aggregates of identical shapes, such as symmetric spiky combinations of spheres and ellipsoids (Malinouskaya et al., 2009; Thies-Weesie et al., 1995), while in reality shapes of each sand are not identical.

Particle shape can be described at three distinctive yet correlated length scales, namely, aspect ratio for particle size, roundness for local corner, and the finest roughness (Barrett, 1980). Dimensionless shape parameters have also been defined across these three scales. Castillo et al. (2015) experimentally estimated $C_{\mathrm{K}-\mathrm{C}}$ of two types of super-ellipsoid-like cubes, i.e., solid hematite cubes and hollow microporous silica cubes, of which sizes and shapes are similar, but roughness features are different. They found that $C_{\mathrm{K}-\mathrm{C}}$ of rough microporous cubes was about $12 \%$

96

97 higher, revealing the notable effects of microscopic roughness on it. With the development of optical equipment for high-resolution 3D surficial data, finding a shape index existing in more98 or full-length morphology is possible. Fractal dimension, accompanied by relative roughness, is 
a candidate, which may unify global surface morphology across multiple length scales (Barclay and Buckingham, 2009; Renard et al., 2013).

In this work, the effect of particle shapes on permeability of porous media is experimentally investigated via hydraulic conductivity tests, as done in classical soil mechanics (Craig, 2004). Realistic particles with controlled fractal dimension are generated using Spherical harmonics (Wei et al., 2020), which are then printed using 3D printers. A modified KozenyCarman equation is proposed by incorporating the fractal dimension and relative roughness, two key morphological parameters for a wide range of geomaterials. Through comparing the model with experimental results and data from literature, we then assess the ability of the proposed model to capture the influence of particle shapes on permeability of granular materials.

\section{Spherical harmonic reconstruction}

\subsection{Reconstruction of natural grains}

For spherical surfaces, through 3D Fourier Transformation, any function set on the surface can be represented as a sum of Spherical Harmonic (SH) function, $Y_{n}^{m}(\theta, \varphi)$, for its orthogonality. SH function has been dedicated to many scientific applications, such as representing orbital configurations (Flügge, 2012), computing physical fields (Turcotte, 1987), and modelling 3D images (Garboczi, 2002). Here, we implement its definition in quantum mechanics to reconstruct star-like surfaces of natural grains via approximating its cumulative radial distance, $r_{I}\left(x_{I}(\theta, \varphi), y_{I}(\theta, \varphi), z_{I}(\theta, \varphi)\right)=r_{I}(\theta, \varphi)=\sqrt{\left(x_{I}-x_{0}\right)^{2}+\left(y_{I}-y_{0}\right)^{2}+\left(z_{I}-z_{0}\right)^{2}}$, between surface points and its centroid, $\left(x_{0}(\theta, \varphi), y_{0}(\theta, \varphi), z_{0}(\theta, \varphi)\right)$, as a function of latitudinal $(\theta \in$ $[0, \pi])$ and longitudinal $(\varphi \in[0,2 \pi))$ coordinates in polar coordinate system:

$r_{I}(\theta, \varphi)=\sum_{n=0}^{\infty} \sum_{m=-n}^{n} c_{n}^{m} Y_{n}^{m}(\theta, \varphi)$,

where $I$ denotes the $I$-th point on particle surface and $c_{n}^{m}$ are the SH coefficients of degree $n$ and order $m$. The process to calculate complex $c_{n}^{m}$ is in S1. Fig. 1 (a) shows nine types of real granular materials of wide-scoped sizes, and the scaling exponential relations in Fig. 1 (b) read

$D_{n} \propto n^{\beta},(n=2,3,4,5 \ldots)$,

where $D_{n}$ is the SH descriptor defined as normalised $L 2 \operatorname{norm}\left(D_{n}=\frac{\sqrt{\sum_{m=-n}^{n}\left\|c_{n}^{m}\right\|^{2}}}{c_{0}}\right), \beta$ is the slope of the regression plot of $\log \left(D_{n}\right)$ versus $\log (n)$. Consequently, following Russ (1994) and Quevedo et al. (2008), $D_{n}$ can be expressed in terms of SH fractal dimension $\left(D_{f}\right)$ :

$D_{n}=D_{2} \cdot\left(\frac{n}{2}\right)^{2 D_{f}-6}$.

According to the Parseval formula, the mean square distance $\left(M_{S D}\right)$ between two SH surfaces with SH coefficient $c_{1, n}^{m}$ and $c_{2, n}^{m}$ can be directly computed (Gerig, 2001): $M_{S D}=\frac{1}{4 \pi} \sum_{n=0}^{\infty} \sum_{m=-n}^{n}\left\|c_{1, n}^{m}-c_{2, n}^{m}\right\|^{2}$. Then, the relative roughness $\left(R_{r}\right)$, quantifying how the irregular particle surface is globally different from its $c_{0}$-determined sphere, can be defined based on $\sqrt{M_{S D}}$ : 
$R_{r}=\frac{\sqrt{\frac{1}{4 \pi} \sum_{n=2}^{n_{\max }} \sum_{m=-n}^{n}\left\|c_{n}^{m}\right\|^{2}}}{c_{0}^{0} \cdot Y_{0}^{0}(\theta, \varphi)}=\sqrt{\sum_{n=2}^{n_{\max }}\left(D_{2} \cdot\left(\frac{n}{2}\right)^{2 D_{f}-6}\right)^{2}}$.

Details of derivation and determination of $D_{f}$ and $R_{r}$ are provided in S2. S3 is referred to for the method of generating randomly shaped particles of given $D_{2}$ and $D_{f}$ via stochastic $c_{n}^{m}$. It 139 has been confirmed that virtual and real particle shapes can have nearly identical shape parameters (e.g., the difference between their mean parameters are all within $2 \%$ ), as long as their corresponding $D_{2}$ and $D_{f}$ are the same (Wei et al., 2018).

(a)

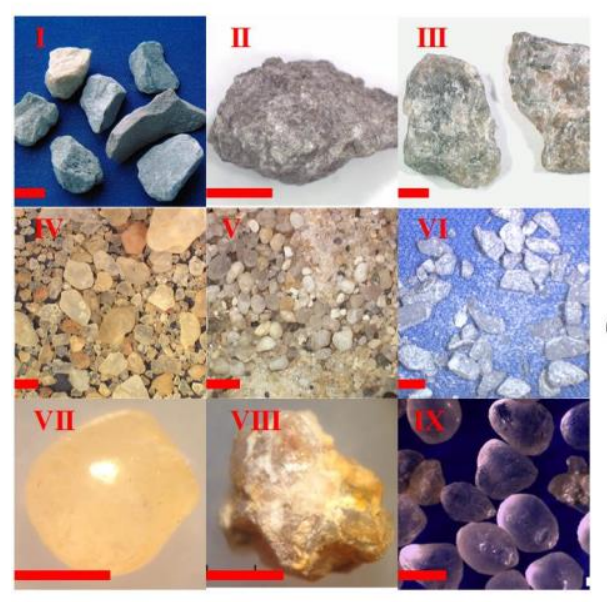

(b)

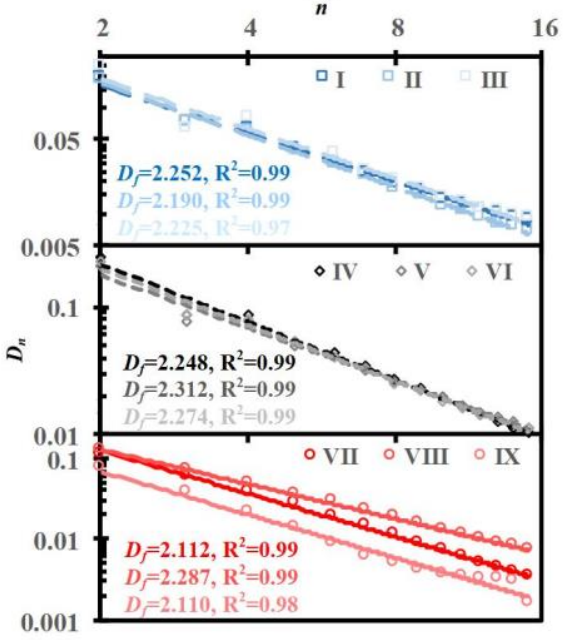

(c)

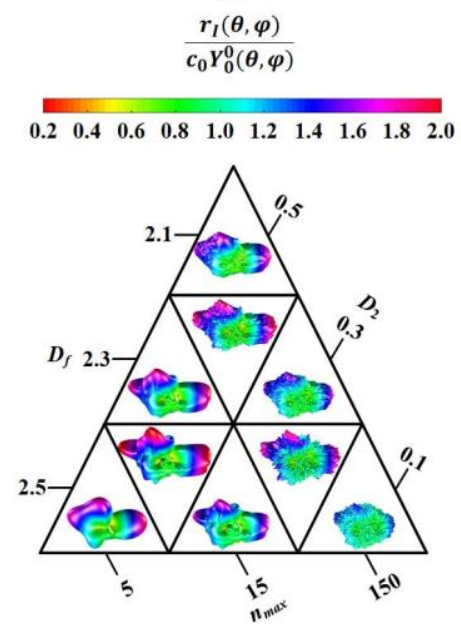

143

\section{4}

145

146

147

Figure 1. The framework from experimentally scanned grains to virtually generated particle shapes. (a) Nine types of real aggregates with scale bar being $1 \mathrm{~cm}$ for I to III and $1 \mathrm{~mm}$ for IV to IX. According to American Society for Testing and Materials (ASTM), the corresponding names of aggregates from I to VI are MA106A-1, MA107-6, MA114F-3, MA111-7, MA99BC-5 and MA106B-4. Images are obtained from an open source software, Virtual Cement and Concrete Testing Laboratory (VCCTL, Bullard, 2014); VII and VIII, Leighton Buzzard sand and highly decomposed granite are from Wei et al. (2018); IX, Ottawa sand, is from Erdoğan et al. (2017). (b) Relations between average $D_{n}$ and $n$ in $\log$-log scales for grains in (a). (c) Virtual representation of generated particle shapes using various maximum SH degree, $n_{\max }, D_{f}$ and $D_{2}$. The colour bar represents the ratio of normalized radial distance to radius of its $c_{0}$-determined sphere.

\subsection{Approximation of volume and surface area}

Since the surface can be implicitly approximated by SH expansion in Eq. (1), grain volume $(V)$ and surface area $(S)$, directly relevant to $S_{0}$ in Kozeny-Carman equation, can be computed and denoted by $c_{n}^{m}$. For SH-generated surface of maximum SH degree, $n_{\max }$, one particle has $\left(n_{\max }+1\right)^{2}$ complex numbers. We set $n_{\max }$ to 15 , which is sufficient in depicting morphology features finer than roundness-length scale of $n_{\max }=8$ (Zhao et al., 2018). Notably, as shown in Figure 1 (c), the applied $n_{\max }$ scales for depicting rougher grain morphology. Via 
163 comparing the surface area between SH-approximated and CT (computer tomography)-based 164 grain shapes, Zhou et al. (2017) found the approximate $n_{\max }$ for rough HDG particles are higher 165 than smooth LBS particles. Computational wise, it is expected there is always space to improve, 166 so $n_{\max }$ can be higher. However, the length-scale of input morphology features is limited by 3D 167 printing resolution. Further reasons for selecting $n_{\max }=15$ as the cut-off length to calculate $S_{0}$ 168 can be found in Sections 3 and 4.

Considering the volume element in polar coordinate system, $d V=r \sin \theta \mathrm{d} \theta \cdot r \mathrm{~d} \varphi \cdot \mathrm{d} r, V$ can be rigidly determined,

$171 V=\int_{0}^{r(\theta, \varphi)} \int_{0}^{\pi} \int_{0}^{2 \pi} r(\theta, \varphi)^{2} \sin \theta \mathrm{d} \varphi \mathrm{d} \theta \mathrm{d} r$.

172 By inserting Eq. (2) into Eq. (6),

$V=\underbrace{\frac{c_{0}{ }^{3}}{6 \sqrt{\pi}}}_{V_{n=0}}+\underbrace{\frac{3 c_{0}}{6 \sqrt{\pi}} \cdot \sum_{n=2}^{n_{\max }}\left(D_{2} \cdot\left(\frac{n}{2}\right)^{2 D_{f}-6}\right)^{2}}_{\Delta V}=\frac{c_{0}{ }^{3}}{6 \sqrt{\pi}}\left(1+3 R_{r}{ }^{2}\right)$,

174 where $V_{n=0}$ is the volume of its $c_{0}$-determined sphere with radius, $c_{0}^{0} \cdot Y_{0}^{0}(\theta, \varphi)=\frac{c_{0}^{0}}{2 \sqrt{\pi}}, \Delta V$ is the changed volume of $V$. To check the integration accuracy of Eq. (6), 2,000 virtual shapes are generated for various sets of $D_{f}$ and $R_{r}$, and the mean $\Delta V\left(\mu_{\Delta V}\right)$ can be calculated. It is found that the ratios of standard deviation to its corresponding $\mu_{\Delta V}$ are less than 0.05 . From figs. 2 (a) and (b), $V$ does depend on $D_{f}$, while the dependence can be entirely caught by $R_{r}$, indicating the reasonableness of Eq. (7).

A surface element on curved surface is based on its local differential properties in Cartesian coordinate system:

$\left|X_{\theta} \times X_{\varphi}\right| d \theta d \varphi$

183 where $\vec{X}=(x, y, z)$ is the surface normal vector, and subscripts denote partial differential items.

184 The unit surface normal vector is $\frac{\overrightarrow{X_{\theta}} \times \overrightarrow{X_{\varphi}}}{\left|\overrightarrow{X_{\theta}} \times \overrightarrow{X_{\varphi}}\right|}$, the components of which are expanded in S4.

185 Accordingly, the analytical solution of SH-generated surface area is

$S=\int_{0}^{\pi} \int_{0}^{2 \pi} r \cdot \sqrt{\left(r_{\varphi}^{2}+r_{\theta}^{2}(\sin \theta)^{2}+r^{2}(\sin \theta)^{2}\right)} \mathrm{d} \varphi \mathrm{d} \theta$,

187 where

188

$r_{\theta}=-\sum_{n=0}^{\infty} \sum_{m=-n}^{n} \sqrt{\frac{(2 n+1)(n-m) !}{4 \pi(n+m) !}} \frac{c_{n}^{m}}{\sin \theta}\left[(n+1) \cos \theta P_{n}^{m}+(m-n-1) P_{n+1}^{m}\right] e^{i m \varphi}$,

and

$r_{\varphi}=\sum_{n=0}^{\infty} \sum_{m=-n}^{n} m \cdot i \cdot c_{n}^{m} Y_{n}^{m}(\theta, \varphi)$.

Although in Eq. (9) a closed-from expression of $S$ is derived, the representation is too complicated to directly relate to $D_{f}$ and $R_{r}$. Similar to the treatment with $V, S$ is split into two parts: $S=S_{n=0}+\Delta S$. From Fig. 2 (c) and Fig. S1, power laws between the mean $\Delta S\left(\mu_{\Delta S}\right), D_{f}$ and $R_{r}$ can be clearly seen. As a simplification, regression analysis is used to define $S$ as a combined function of the two, 
$S=\underbrace{c_{0}^{2}}_{S_{n=0}}+\underbrace{c_{0}^{2} \cdot \frac{\pi}{20} \cdot R_{r^{2}}^{\frac{\pi}{2}} \cdot D_{f}^{3.874}}_{\Delta S}=c_{0}^{2}\left(1+\frac{\pi}{20} \cdot R_{r^{\frac{\pi}{2}}}{ }^{2} D_{f}^{3.874}\right)$.

197

198

199

200

201

202

Fig. 2 (d) indicates the efficiency of Eq. (12) in approximating $S$. Notably, the ranges of $R_{r}$ or $D_{2}$ and $D_{f}\left(D_{2} \in[0,0.3], D_{f} \in[2,2.6]\right)$, in which the regression is conducted, are much larger than those of real sands, as in Fig. 1 (b). Considering the high value of R-square, it is reasonable to directly apply Eq. (12) to approximate surface area of SH-generated surfaces in subsequent studies.

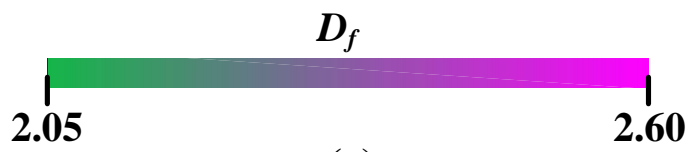

(a)

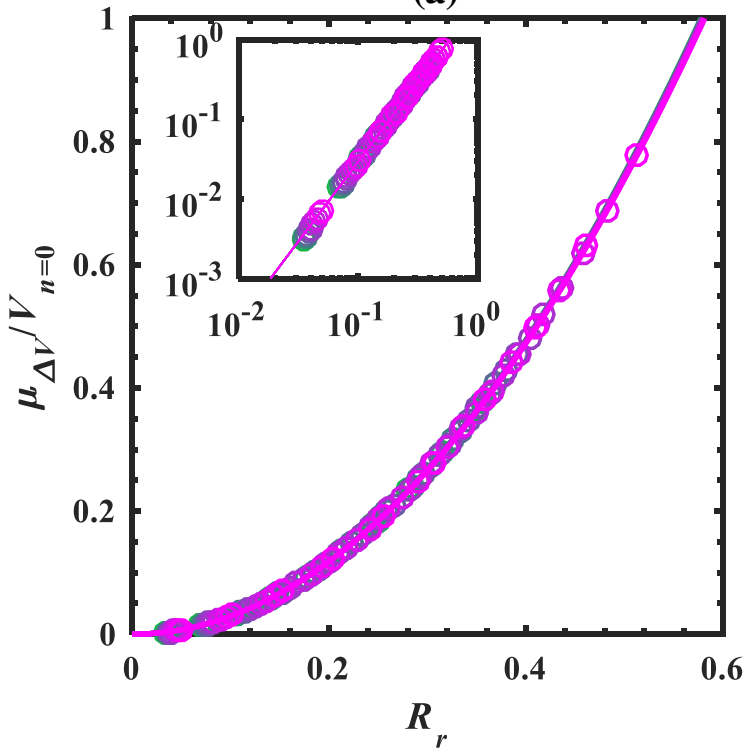

(c)

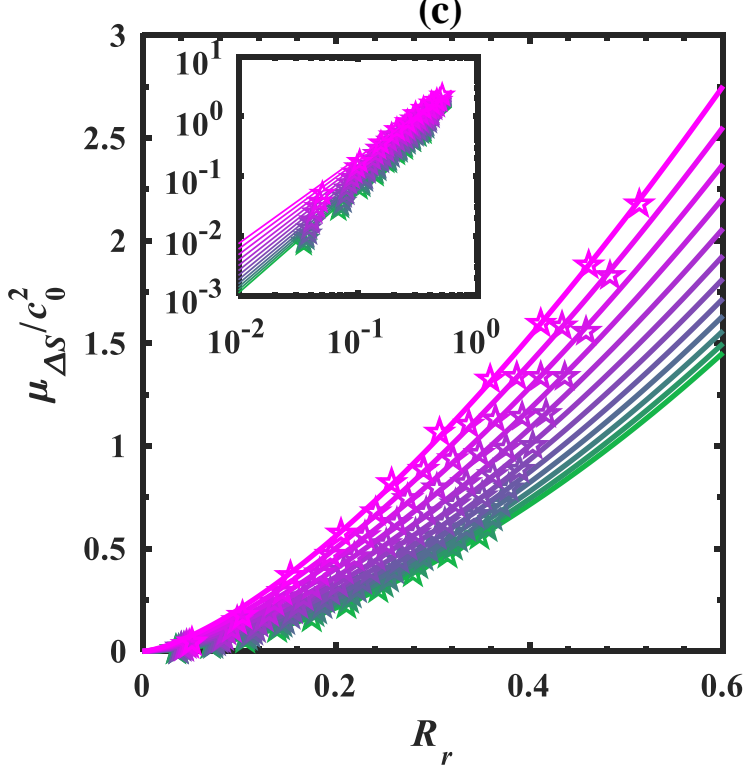

(b)

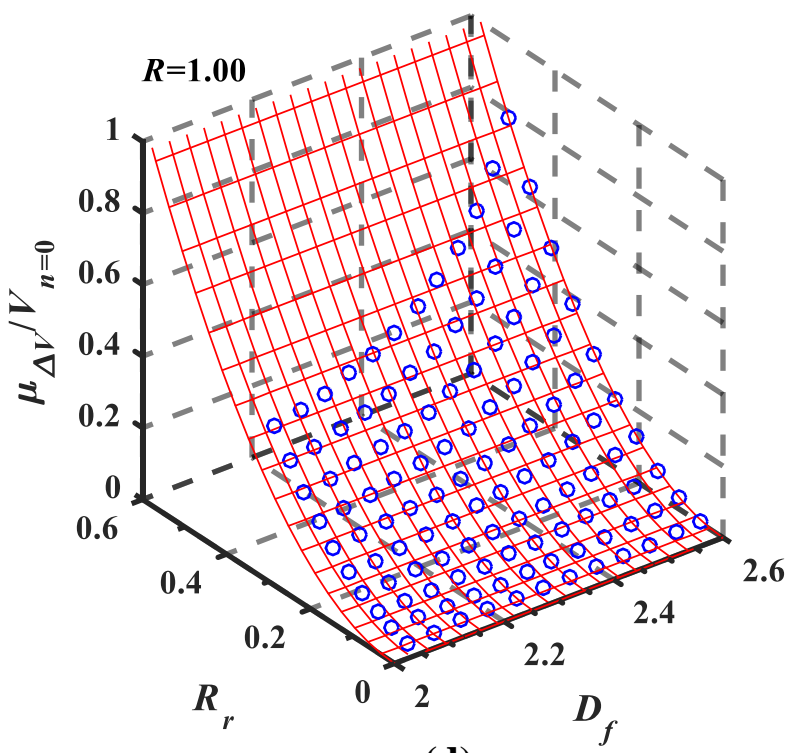

(d)

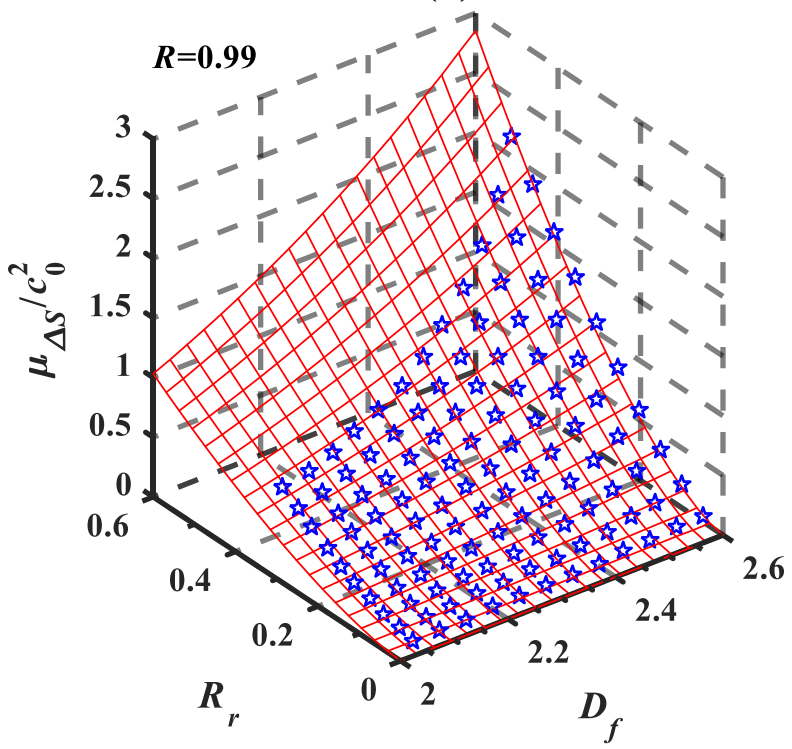

203 
204 Figure 2. Influences of $D_{f}$ and $R_{r}$ on mean varied $V$ and $S$, of which every data point is from 2,000 virtual particles of $n_{\max }=15$. The morphology features smaller than cut-off length does not contribute to $S_{0}$. $\mathrm{O}$ and denote $\mu_{\Delta S}$ and $\mu_{\Delta V}$ from closed form solutions from Eq. (6) or Eq. (9). The solid line and mesh in (a) and (b) denote solutions from Eq. (7), while the solid line and mesh in (c) and (d) are from regression analysis. For every data point of $\mu_{\Delta S}$ and $\mu_{\Delta V}$, the ratio of its standard deviation to itself is less than 0.05. The efficiency of the developed regression conducted to approximate $\mu_{\Delta S}$ depends on the number of training data. It is found that when the number of particle shapes for every point data in (d) is larger than 2000 , the resulted parameters in Eq. (12) vary less than $1 \%$.

\section{3D printed grains and water permeability experiments}

In recent years, for the capability of producing particles with controlled morphology and material properties, 3D printing (Jiang et al., 2020) are becoming popular in studies of granular mechanics (Miskin and Jaeger, 2013; Ju et al., 2018; Gupta et al., 2019) and hydraulic conductivity (Suzuki et al., 2017; Fang et al., 2018; Adamidis et al., 2019). However, many studies just consider regular aspherical shapes for idealization (Athanassiadis et al., 2014; Murphy et al., 2019). Meanwhile, for irregular shapes most 3D printed particles are produced as one-to-one of CT data for estimating whether they can capture real granular behaviour. As pointed by the pioneering work on 3D printable geomaterials (Hanaor et al., 2016), except for printing resolution and materials, progress can be dedicated to geometrical model of printed grains. The following advantages can be obtained: i) input morphological and statistical parameters are extracted from real ones; ii) as many as desired realistic particle morphology features are depicted, such as coexistence of smoothed faces, angularity and branching; iii) ready to study effects induced by varying morphology. Looking back to Fig. 1, all of the mentioned advantages can be achieved via our proposed $\mathrm{SH}$ framework.

In this work, a poly-jet layer-printing 3D printer (Objet Eden 250) with horizontal and vertical resolutions of $4 \mu \mathrm{m}$ and $32 \mu \mathrm{m}$ was used. For more information on the printer and printing materials, please refer to the relevant study (Hanaor et al., 2016). Each batch containing 2,000 particles, placed by 40 rows and 50 columns in one layer, is printed. As suggested by Adamidis et al. (2020), with the help of 3D printing (the resolutions are $16 \mu \mathrm{m}$ and $40 \mu \mathrm{m}$ in horizontal and vertical resolutions), shape parameters of printed grains of size equal around $2 \mathrm{~mm}$ are within $5 \%$ difference from input geometries, proved by X-Ray CT images. With SH coefficients from section 2.1, we import polar coordinates of icosahedron-based geodesic structure with 1,280 triangular faces into Eq. (2) to generate STereolithography (STL) input files suitable for $3 \mathrm{D}$ printing. The reason why surfaces composed of 1,280 faces are selected is that the triangle edge length should be larger than the printing resolution for printing all given geometry features. Edges connecting adjacent vertices of such surficial meshes have a spherical angle of about 0.14 . The depicted grain morphology is influenced by both the number of vertices on the surface and $n_{\text {max }}$. Considering the relation between a proper angle resolution $(\Delta \theta)$ and any given $n$ (Jekeli, 1996; Jekeli et al., 2007), $\Delta \theta=\frac{\pi}{n}$, and the vertical printing resolution, the finest morphology features, which can be successfully printed, are determined by $n_{\max }$ up to about 30 , since $r_{I}(\theta, \varphi) \approx 1 \mathrm{~mm}$. This being said, there are two main reasons why $n_{\max }=15$ is 246 selected: i) when removing supporting wax after printing, $\mathrm{NaOH}$ solution may corrode finer 
grain surface features; ii) for $D_{f}<2.75$, when $n_{\max }=15$, in S6 it is proved that $R_{r}$ depending $S_{0}$ has converged and is less than $\frac{2^{6-2 D_{f}} \cdot D_{2}}{\sqrt{11-4 D_{f}}}$. Furthermore, we align the longest particle dimension with vertical direction to optimally exploit the printing resolution.

Fig. 3 (a)-(d) illustrates the cumulative distributions of input shape indices for four groups of particles with the same $c_{0}=3.5 \mathrm{~mm}$. The non-uniformly shaped particles have $D_{2}=0.1$, close to those of fine aggregates in Fig. 1 (a) and (b). It can be seen that the printed particles have uniform gradings (Fig. 3(a)), and their sphericity is also uniform within a group, consistent with Eqs. (5), (7), and (12). The variation in aspect ratio indicates the efficiency of the proposed SH framework in generating macroscopically different particle shapes yet with statistically similar finer morphological features, which is further proved in Fig. 3 (d) by the median-sized feature, roundness. After finishing printing, the printed grains with supporting wax were kept in a bath with aqueous solution containing $2 \% \mathrm{NaOH}$ for 30 minutes. Then, the grains were manually rubbed in the basin to remove remaining wax, and finally rinsed with pure water. At last, wetted grains were dried overnight at constant room temperature of $20^{\circ} \mathrm{C}$, as in Fig. 3 (e).
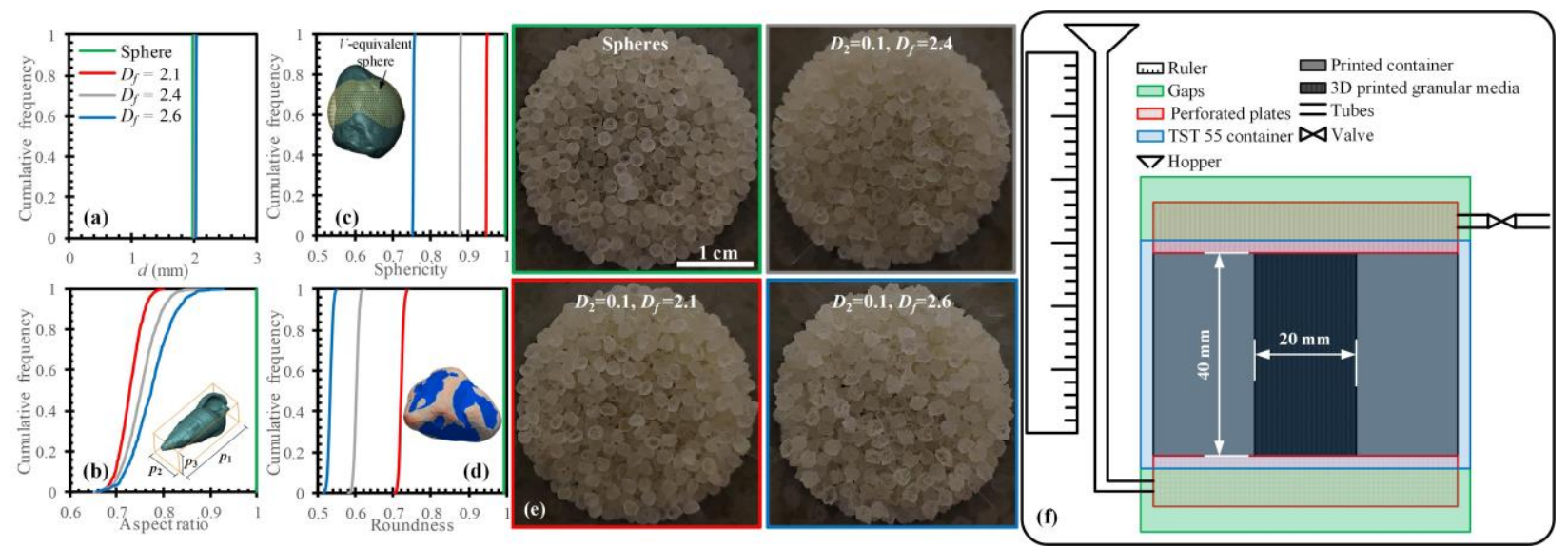

Figure 3. (a)-(d) Cumulative distributions of classical shape indices of printed grains of $c_{0}=3.5$ $\mathrm{mm}(d \approx 2 \mathrm{~mm})$ and $D_{2}=0.1$. Each group contains about 10,000 particles. (a) Equivalent-sphere diameter. (b) Aspect ratio, defined as $A_{r}=E_{i} \times F_{i}$, with mean elongation and flatness $E_{i}=$ $p_{2} / p_{1}$ and $F_{i}=p_{3} / p_{2}$, respectively, where $p_{1}, p_{2}$ and $p_{3}$ are the particle's principal dimensions calculated by principal component analysis. (c) Sphericity $S_{p}=\sqrt[3]{36 \pi V^{2}} / S$ reflects the deviation of surface area from its volume-equivalent sphere. Notably, $S_{p}$ can be used to indicate how much error can be made if the area of grain volume-equivalent sphere is considered to approximate its surface area. For example, if the area of volume-equivalent sphere is applied, about $25 \%$ error can be induced. (d) Roundness $R_{M}=\sum\left(\mathbb{S}_{l} \cdot \frac{k_{\text {in }}}{k_{M, l}}\right) / \sum \mathbb{S}_{l}, \forall k_{M, l} \leq k_{\text {in }}$, where $\mathbb{S}_{l}$ is the area of $l$-th triangle, of which the mean median curvature value of its three vertices is $k_{M, l}$, and $k_{\text {in }}$ is the curvature value of maximum inscribed sphere of the particle. The colour in (d) 
Capabilities of one-to-one printed sands to reproduce hydraulic-related features have been comprehensively demonstrated by Adamidis et al (2020). The standard TST-55 permeameter (GB/T 9357-2008) was used for water permeability tests via falling-head method. Since the volume of original container of TST-55 permeameter is too large to be completely filled with our printed grains, a hollow cylinder was printed as a filler to reduce the effective volume of the original container (Fig. 3(f)). The ratio of average grain diameter to container width is about 0.1 , which is sufficiently small to obtain representative measurements of hydraulic conductivity (Garcia et al., 2009). For permeability test, grains were poured into the container, and the excessive grains were carefully levelled off. To vary the porosity, different intensity of tapping and compression were applied during pouring. As pointed out by Chapuis (2012), errors in laboratory tests of the hydraulic conductivity mainly fall into two categories: i) the occurrence of preferential leakage between the porous specimen and the rigid permeameter wall; ii) the full saturation of the porous media is not achieved. To avoid the former, the inner surface of the printed hollow cylinder is mapped by closely wounded coils with sectional circles of diameter equal to $1 \mathrm{~mm}$. For the latter, the permeameter was vibrated during first several cycles of water injection until no observable bubbles can be seen. By slowly seeping the fluid from the base to the top, the gravity is also in favour of removing the air. In Taylor et al. (2017), hydraulic conductivity of natural LBS particles is conducted by seepage from the base, of which the results are also compared with samples with de-air process. It was found the difference between the two is within $2 \%$. For subsequent studies, the application of degassed water is a good choice. Via repeating the tests for a given experiment, it is found that the difference of measured coefficients of permeability is also within $2 \%$.

Videos at $30 \mathrm{fps}$ were recorded during permeability tests. Compared with classical

$k=\frac{a \cdot l}{A \cdot\left(t_{n+1}-t_{n}\right)} \cdot \ln \frac{h_{n}}{h_{n+1}}$,

then we modify it as

$k_{n}=\frac{a \cdot l}{A} \cdot \frac{\ln h_{n}-\ln h_{n+1}}{t_{n+1}-t_{n}}$,

where $a$ and $A$ are the areas of the tube and the porous sample cross-section, respectively, and $n$ is the frame number of the video. By using all the data during each permeability test (typically several hundreds of frames), $k$ can be determined by fitting the slopes in $(\ln h)-t$ curves. Seven representative experiments were conducted for each group of grain type at each porosity, and the invariance to mean ratio is less than $5 \%$, indicating good repeatability of experiments.

\section{Results and modified Kozeny-Carman equation}

Fig. 4 (a) shows the measured absolute coefficients of water permeability, $K$, as a function of porosity $\varepsilon$ for different particle shapes. With the increase of $D_{f}$, the tortuosity of pores in porous media of same $\varepsilon$ becomes higher, resulting in lower $K$. Some studies have revised Kozeny-Carman equation based on the specific grading to approximate pore diameter, such as in Chapuis (2004) with $k=2.4622 \cdot\left[D_{10}{ }^{2} \cdot \frac{\varepsilon^{3}}{(1-\varepsilon)^{2}}\right]^{0.7825}$, where $D_{10}$ is the $10 \%$ finer particle size. 
316 This approach is not applicable to the current work, because the gradings of our four types of 317 printed grains are deemed equal, as shown in Fig. 3 (a).

Since printed grains are reconstructed along vertices of 1280-triangle based icosahedron geodesic structures, $S$ and $V$ can be directly calculated and the corresponding $S_{0}$ can be readily obtained. Fig. 4 (b) compares $C_{\mathrm{K}-\mathrm{C}}$ calculated by importing measured permeability into Eq. (1). It is surprising that the shape factor, $C_{\mathrm{K}-\mathrm{C}}$, is insensitive to particle shapes from spheres to highly irregular shapes with $D_{f}=2.6$. However, if $S_{0}$ is approximated based on volume-equivalent spheres, an increase in $C_{\mathrm{K}-\mathrm{C}}$ is observed for irregular particles. Here, we propose that the increase is the manifestation of the errors from calculation of $S_{0}$. The value of $C_{\mathrm{K}-\mathrm{C}}$ of printed spheres is about 5.5, within the relevant experimental values $5.0 \sim 6.4$ for mono-sized spheres (Carman, 1956). This consistency in $C_{\mathrm{K}-\mathrm{C}}$ of mono-sized spheres proves the adequacy of our experiments to measure permeability.

Via electrokinetic analysis of complex anion adsorption, Hanaor et al. (2014) concluded that particle surface area is unlimited. If so, $K$ would approach to zero, which is certainly problematic. According to Koch and Brady (1985) and Durlofsky and Brady (1987), the decay length, called Brinkman screening length, of viscous flow velocity disturbance in fixed spherical granular porous media roughly equals $\sqrt{K}$. This decay length can be regarded as the cut-off length for effects of surface roughness on the near flow field, below which surface features will have limited effect on the permeability (Castillo et al., 2015). Thus, only morphological features of length beyond such length is considered into $S_{0}$. Note that the roughness of printed grains is deemed the same and the 3D printer resolution is $32 \mu \mathrm{m}$, which is in the same order of magnitude as mean triangular edge length $(\approx 136 \mu \mathrm{m})$ of printed triangle-based grains, as well as the decay length $(\approx 20 \mu \mathrm{m})$. We fit data points of printed spheres in Fig. 4 (b) to get $C_{\mathrm{K}-\mathrm{C}}$ for the roughness generated by the printer. Substituting Eqs. (7) and (12) into Eq. (1), the modified KozenyCarman equation is

$$
K=k \cdot \frac{\mu}{\gamma}=\frac{1}{5.5} \frac{c_{0}{ }^{2}\left(1+3 R_{r}^{2}\right)^{2}}{36 \pi\left(1+\frac{\pi}{20} \cdot R_{r}^{\frac{\pi}{2}} \cdot D_{f}^{3.874}\right)^{2}} \frac{\varepsilon^{3}}{(1-\varepsilon)^{2}} \text {. }
$$

Notably, $R_{r}$ is dependent on $n_{\max }$, which can be related to the decay length with $\sqrt{K} \propto$

$\frac{\pi}{n_{\max }} r_{I}(\theta, \varphi) \approx \frac{\pi}{n_{\max }} c_{0}^{0} Y_{0}^{0}(\theta, \varphi)$,

$n_{\max } \propto \frac{c_{0}}{2} \cdot \sqrt{\frac{\pi}{K}}$

Return to Eq. (5), the value of $R_{r}$ converges and is less than $\frac{2^{6-2 D_{f}} \cdot D_{2}}{\sqrt{11-4 D_{f}}}$. In addition, from Fig. S2 when $n_{\max }$ is up to 15 , the value of $R_{r}$ is very close to the convergence. Hence, we choose $n_{\max }=$ 15 to calculate $R_{r}$. After importing $R_{r}$ calculated from Eq. (5), $c_{0}$, and $D_{f}$ into Eq. (15), predictions of $K$ can be obtained, which agree well with the experimental results in Fig. 4 (c). It proves the reasonableness of $n_{\max }=15$, as a cut-off, to calculate $S_{0}$ for $K$, at a low Reynolds number. Note this modified equation does not contain any additional fitting parameters and only requires porosity and morphological parameters accessible from actual grain shapes.

To further check applicability of Eq. (15) for natural grains, 3D surficial data (e.g., XRay CT data) are needed for determination of $c_{0}, R_{r}, D_{f}$ at $n_{\max }=15$. Two types of poorly graded natural quartz sands (LBS from Taylor et al. (2017) and Ottawa sand from Schroth et al. (1996)) are taken into consideration in Figs. 4 (c) and (d), where $R_{r}$ and $D_{f}$ are calculated from Fig. 1 (b), 
sample VII and IX, respectively. Although there is no perfectly uniform grading of the two sands, predicted values are close to experimental values. The model validated by data of natural sands appears to be appropriate over three orders of magnitudes of water permeability coefficient. It is possible that the proposed equation is not appropriate for well graded grains, because packing structures or pore distributions can significantly differ from those of uniform gradings. How grain grading distribution affects the validity of the equation deserves future studies. In this study, only statistical morphological features $\left(R_{r}\right.$ and $\left.D_{f}\right)$ and volume are uniform, but other shape parameters lay in a wide range, as in Fig. 3 (b), which could also alter pore structures. This may explain why the proposed model can hold for $C_{u}$ (grading uniform coefficient) up to 1.5 . To the best knowledge of the authors, it is the first time to experimentally study permeability coefficients of non-spherical uniform grains with focus on whether the shape coefficient in Kozeny-Carman equation is dependent on grain shapes, when effects of roughness are isolated. Surprisingly, the Kozeny-Carman shape coefficient is insensitive to particle shapes, contrary to what has been widely suggested before. Although via hydraulic conductivity tests such insensitivity is found, how much it can be generalized to other types of porous media with global multi-scale morphology features, such as fibrous structures, needs further research.
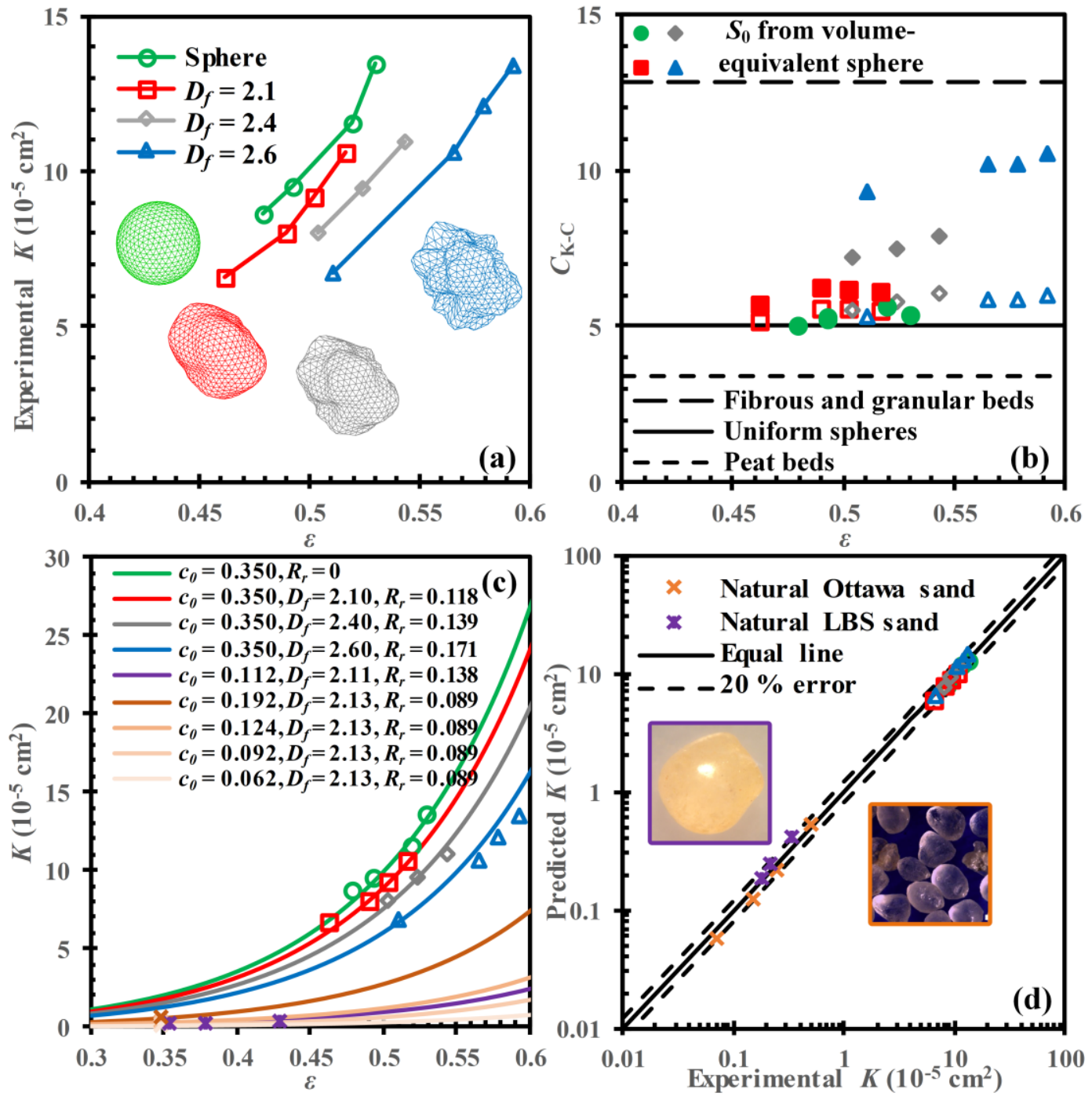
374 Figure 4. (a) Experimental water permeability coefficients as a function of porosity. The snapshots represent 1280-face particle surface with the same polar coordinates of icosahedronbased geodesic surface with the same number of facets. (b) Relations between $C_{\mathrm{K}-\mathrm{C}}$ and porosity for $S_{0}$ calculated from STLs (void symbols) or volume-equivalent spheres (solid symbols). Values for fibrous and granular beds, uniform spheres, and peat beds are from $\mathrm{Li}$ and $\mathrm{Gu}(2005)$, Carman (1937), and Mathvan and Viraraghavan (1992), respectively. (c) Relations between porosity and water permeability coefficients with lines for the proposed equation and data points for experimental data. The colour orange and purple denote natural Ottawa sand and LBS particles. The unit of $c_{0}$ is $\mathrm{cm}$. (d) Comparisons between experimental results and predictions of modified Kozeny-Carman equation, including two natural LBS (Taylor et al., 2017) and Ottawa sand (Schroth et al., 1996) particles.

\section{Conclusions}

With the help of 3D printing, at low Reynold's number the present work explores coefficients of permeability of uniformly graded irregular grains with controlled shapes and fractal morphological features. The results indicate that particle shape does influence permeability coefficients; that is, with the increase of fractal dimension only above a moderate roughness length scale, induced high tortuosity can slow water seeping through granular porous media of the same porosity. A modified Kozeny-Carman equation is proposed by incorporating the specific surface area calculated based on spherical harmonics, where it is derived as a function of particle size, relative roughness and fractal dimension. It is found that the shape coefficient in Kozeny-Carman equation is insensitive to particle shapes, ranging from spheres to significantly irregular shapes of high fractal dimension equal to 2.6. We also check the applicability of the proposed model on poorly graded natural grains by comparing with data in the literature. Good agreements are observed. The current work provides the first experimental study on permeability of uniformly graded aspherical grains with controlled particle shapes and fractal morphological features, and advances the understanding of their correlations. Extensive research on how the combined effects of grain sizes and shapes affect the proposed permeability model prediction should be performed in future studies. To better solve this outstanding issue, appropriately quantifying stochasticity of tortuosity, which is for pore structures that fluids flow through, may be necessary in deterministically predictive models of permeability coefficients.

\section{Acknowledgments, Samples, and Data}

We thank Dr. Junxing Zheng and Dr. Quan Sun from Iowa State University for providing us relations between $D_{n}$ and $n$ in Fig. 1 (b). We thank Dr. Bowei Yu from the University of Sydney for help and guidance in experimental set-ups. Dr. Behzad Ghanbarian from Kansas State University and Dr. Budi Zhao from University College Dublin are thanked for leading us to the papers, Schroth et al. (1996) and Taylor et al. (2017). Fruit comments, which enhances our research quality significantly, from two reviewers are also greatly acknowledged. The data and codes relevant to this study can be found in http://doi.org/10.5281/zenodo.4387218. 


\section{References}

Adamidis, O., Alber, S., \& Anastasopoulos, I. (2020). Assessment of Three-Dimensional Printing of Granular Media for Geotechnical Applications. Geotechnical Testing Journal, 43(3).

Athanassiadis, A. G., Miskin, M., Kaplan, P., Rodenberg, N., Lee, S. H., Merritt, J., ... \& Jaeger, H. M. (2014). Particle shape effects on the stress response of granular packings. Soft Matter, 10(1), 48-59.

Barclay, D. R., \& Buckingham, M. J. (2009). On the shapes of natural sand grains. Journal of Geophysical Research Solid Earth, 114(B2).

Barrett, P. J. (1980). The shape of rock particles, a critical review. Sedimentology, 27(3), 291303.

Bullard, J. W. (2014). Virtual Cement and Concrete Testing Laboratory: Version 9.5 User Guide (No. Special Publication (NIST SP)-1173).

Carman, P. C. (1937). Fluid flow through a granular bed. Transactions of the Institution of Chemical Engineers, 15, 150-167.

Carman, P. C. (1956). Flow of gases through porous media. Butterworths Scientific Publications, London.

Carrier III, W. D. (2003). Goodbye, hazen; hello, kozeny-carman. Journal of Geotechnical and Geoenvironmental Engineering, 129(11), 1054-1056.

Castillo, S. I., Thies-Weesie, D. M., \& Philipse, A. P. (2015). Formation and liquid permeability of dense colloidal cube packings. Physical Review E, 91(2), 022311.

Chapuis, R. P. (2004). Predicting the saturated hydraulic conductivity of sand and gravel using effective diameter and void ratio. Canadian Geotechnical Journal, 41(5), 787-795.

Chapuis, R. P. (2012). Predicting the saturated hydraulic conductivity of soils: a review. Bulletin of Engineering Geology and the Environment, 71(3), 401-434.

Chapuis, R. P., \& Aubertin, M. (2003). On the use of the Kozeny Carman equation to predict the hydraulic conductivity of soils. Canadian Geotechnical Journal, 40(3), 616-628.

Chikhi, N., Coindreau, O., Li, L. X., Ma, W. M., Taivassalo, V., Takasuo, E., ... \& Laurien, E. (2014). Evaluation of an effective diameter to study quenching and dry-out of complex debris bed. Annals of Nuclear Energy, 74, 24-41.

Costa, A. (2006). Permeability-porosity relationship: A reexamination of the Kozeny-Carman equation based on a fractal pore-space geometry assumption. Geophysical Research Letters, 33(2).

Craig, R. F. (2004). Craig's soil mechanics. CRC press.

David, C. (1993). Geometry of flow paths for fluid transport in rocks. Journal of Geophysical Research: Solid Earth, 98(B7), 12267-12278.

Durlofsky, L. J., \& Brady, J. F. (1987). Analysis of the Brinkman equation as a model for flow in porous media. Physics of Fluids, 30(11), 3329-3341. 
Erdoğan, S. T., Forster, A. M., Stutzman, P. E., \& Garboczi, E. J. (2017). Particle-based characterization of Ottawa sand: shape, size, mineralogy, and elastic moduli. Cement and Concrete Composites, 83, 36-44.

Fang, Y., Elsworth, D., Ishibashi, T., \& Zhang, F. (2018). Permeability evolution and frictional stability of fabricated fractures with specified roughness. Journal of Geophysical Research: Solid Earth, 123(11), 9355-9375.

Feng, S., Vardanega, P. J., Ibraim, E., Widyatmoko, I., \& Ojum, C. (2019). Permeability assessment of some granular mixtures. Géotechnique, 69(7), 646-654.

Flügge, S. (2012). Practical quantum mechanics. Springer Science \& Business Media.

Garboczi, E. J. (2002). Three-dimensional mathematical analysis of particle shape using X-ray tomography and spherical harmonics: Application to aggregates used in concrete. Cement and Concrete Research, 32(10), 1621-1638.

Garcia, X., Akanji, L. T., Blunt, M. J., Matthai, S. K., \& Latham, J. P. (2009). Numerical study of the effects of particle shape and polydispersity on permeability. Physical Review E, $80(2), 021304$.

GB/T 9357-2008, Instrument for soil test-Permeameter. (2008). General Administration of Quality Supervision, Inspection and Quarantine of the People's Republic of China \& Standardization Administration. (2008). Retrieved from http://openstd.samr.gov.cn/bzgk/gb/newGbInfo?hcno=902BACD0C9CB814080656150D D1BD5BA.

Gerig, G., Styner, M., Jones, D., Weinberger, D., \& Lieberman, J. (2001, December). Shape analysis of brain ventricles using spharm. In Proceedings IEEE Workshop on Mathematical Methods in Biomedical Image Analysis (MMBIA 2001) (pp. 171-178). IEEE.

Ghanbarian, B., Hunt, A. G., Ewing, R. P., \& Sahimi, M. (2013). Tortuosity in porous media: a critical review. Soil science society of America journal, 77(5), 1461-1477.

Gupta, R., Salager, S., Wang, K., \& Sun, W. (2019). Open-source support toward validating and falsifying discrete mechanics models using synthetic granular materials-Part I: Experimental tests with particles manufactured by a 3D printer. Acta Geotechnica, 14(4), 923-937.

Hanaor, D. A. H., Ghadiri, M., Chrzanowski, W., \& Gan, Y. (2014). Scalable surface area characterization by electrokinetic analysis of complex anion adsorption. Langmuir, 30(50), 15143-15152.

Hanaor, D. A. H., Gan, Y., Revay, M., Airey, D. W., \& Einav, I. (2016). 3D printable geomaterials. Géotechnique, 66(4), 323-332.

Hazen, A. (1892). Some physical properties of sands and gravels. Mass. State Board of Health. 24th Annual Report, 539-556.

Higdon, J. J., \& Ford, G. D. (1996). Permeability of three-dimensional models of fibrous porous media. Journal of Fluid Mechanics, 308(-1), 341-361. 
Jekeli, C. (1996). Spherical harmonic analysis, aliasing, and filtering. Journal of Geodesy, 70(4), 214-223.

Jekeli, C., Lee, J. K., \& Kwon, J. H. (2007). On the computation and approximation of ultrahigh-degree spherical harmonic series. Journal of Geodesy, 81(9), 603-615.

Jiang, L., Yoon, H., Bobet, A., \& Pyrak-Nolte, L. J. (2020). Mineral fabric as a Hidden Variable in fracture formation in Layered Media. Scientific reports, 10(1), 1-9.

Johnson, D. L., Koplik, J., \& Schwartz, L. M. (1986). New pore-size parameter characterizing transport in porous media. Physical Review Letters, 57(20), 2564.

Ju, Y., Ren, Z., Li, X., Wang, Y., Mao, L., \& Chiang, F. P. (2019). Quantification of hidden whole-field stress inside porous geomaterials via three-dimensional printing and photoelastic testing methods. Journal of Geophysical Research: Solid Earth, 124(6), 5408-5426.

Koch, D. L., \& Brady, J. F. (1985). Dispersion in fixed beds. Journal of Fluid Mechanics, 154, 399-427.

Li, J., \& Gu, Y. (2005). Coalescence of oil-in-water emulsions in fibrous and granular beds. Separation and Purification Technology, 42(1), 1-13.

Malinouskaya, I., Mourzenko, V. V., Thovert, J. F., \& Adler, P. M. (2009). Random packings of spiky particles: Geometry and transport properties. Physical Review E, 80(1), 011304.

Mathavan, G. N., \& Viraraghavan, T. (1992). Coalescence/filtration of an oil-in-water emulsion in a peat bed. Water Research, 26(1), 91-98.

Miskin, Marc Z, \& Jaeger, Heinrich M. (2013). Adapting granular materials through artificial evolution. Nature Materials, 12(4), 326-331.

Mortensen, N. A., Okkels, F., \& Bruus, H. (2005). Reexamination of Hagen-Poiseuille flow: Shape dependence of the hydraulic resistance in microchannels. Physical Review E, 71(5), 057301.

Mollon, G., \& Zhao, J. (2012). Fourier-Voronoi-based generation of realistic samples for discrete modelling of granular materials. Granular matter, 14(5), 621-638.

Murphy, K. A., Dahmen, K. A., \& Jaeger, H. M. (2019). Transforming mesoscale granular plasticity through particle shape. Physical Review X, 9(1).

Philipse, A. P., \& Pathmamanoharan, C. (1993). Liquid permeation (and sedimentation) of dense colloidal hard-sphere packings. Journal of Colloid and Interface Science, 159(1), 96-107.

Quevedo, R., Mendoza, F., Aguilera, J. M., Chanona, J., \& Gutierrezlopez, G. F. (2008). Determination of senescent spotting in banana (Musa cavendish) using fractal texture Fourier image. Journal of Food Engineering, 84(4), 509-515.

Ren, X. W., \& Santamarina, J. C. (2018). The hydraulic conductivity of sediments: A pore size perspective. Engineering Geology, 233, 48-54.

Renard, F., Candela, T., \& Bouchaud, E. (2013). Constant dimensionality of fault roughness from the scale of micro-fractures to the scale of continents. Geophysical Research Letters, 40(1), 83-87. 
Russ, J. C. (1991). Fractal Surfaces. Springer, Berlin: Plenum Press.

Santamarina, J. C., Klein, K. A., Wang, Y. H., \& Prencke, E. (2002). Specific surface: determination and relevance. Canadian Geotechnical Journal, 39(1), 233-241.

Schroth, M. H., Istok, J. D., Ahearn, S. J., \& Selker, J. S. (1996). Characterization of Millersimilar silica sands for laboratory hydrologic studies. Soil Science Society of America Journal, 60(5), 1331-1339.

Seelheim, F. (1880). Methoden zur Bestimmung der Durchlässigkeit des Bodens. Zeitschrift für analytische Chemie, 19(1), 387-418.

Shepherd, R. G. (1989). Correlations of permeability and grain size. Groundwater, 27(5), 633638.

Sun, W., Andrade, J. E., Rudnicki, J. W., \& Eichhubl, P. (2011). Connecting microstructural attributes and permeability from 3D tomographic images of in situ shear-enhanced compaction bands using multiscale computations. Geophysical Research Letters, 38(10).

Suzuki, A., Watanabe, N., Li, K., \& Horne, R. N. (2017). Fracture network created by 3-D printer and its validation using CT images. Water Resources Research, 53(7), 6330-6339.

Thies-Weesie, D. M., Philipse, A. P., \& Kluijtmans, S. G. (1995). Preparation of Sterically Stabilized Silica-Hematite Ellipsoids-Sedimentation, Permeation, and Packing Properties of Prolate Colloids. Journal of Colloid and Interface Science, 174(1), 211-223.

Turcotte, D. L. (1987). A fractal interpretation of topography and geoid spectra on the Earth, Moon, Venus, and Mars. Journal of Geophysical Research: Solid Earth, 92(B4), E597E601.

Taylor, H., Osullivan, C., Sim, W. W., \& Carr, S. J. (2017). Sub-particle-scale investigation of seepage in sands. Soils and Foundations, 57(3), 439-452.

Wang, J. P., François, B., \& Lambert, P. (2017). Equations for hydraulic conductivity estimation from particle size distribution: A dimensional analysis. Water Resources Research, 53(9), 8127-8134.

Wang, C., Elsworth, D., \& Fang, Y. (2019). Ensemble shear strength, stability, and permeability of mixed mineralogy fault gouge recovered from 3D granular models. Journal of Geophysical Research: Solid Earth, 124(1), 425-441.

Wei, D., Wang, J., Nie, J., \& Zhou, B. (2018). Generation of realistic sand particles with fractal nature using an improved spherical harmonic analysis. Computers and Geotechnics, 104, $1-12$.

Wei, D. H., Zhai, C. P., HANAOR, D., \& Gan, Y. X. (2020). Contact behaviour of simulated rough spheres generated with spherical harmonics. International Journal of Solids and Structures, 193, 54-68.

Zheng, J., Ju, Y., \& Wang, M. (2018). Pore-scale modeling of spontaneous imbibition behavior in a complex shale porous structure by pseudopotential lattice Boltzmann method. Journal of Geophysical Research: Solid Earth, 123(11), 9586-9600. 
manuscript submitted to Geophysical Research Letters

569 Zhou, B., Wang, J., \& Wang, H. (2017). Three-dimensional sphericity, roundness and fractal 570 571 dimension of sand particles. Géotechnique, 68(1), 18-30. 